\title{
Oncogenomics: Clinical pathogenesis approach
}

\author{
Gupta G. ${ }^{1}$, Gupta N. ${ }^{2}$, Trivedi S. ${ }^{3}$, Patil P. ${ }^{5}$, Gupta M. ${ }^{2}$, Jhadav A. ${ }^{4}$, Vamsi K.K. ${ }^{6}$, Khairnar Y. \\ Boraste A. ${ }^{4}$, Mujapara A.K. ${ }^{7}$, Joshi B. ${ }^{8}$ \\ ${ }^{1}$ S.D.S.M. College Palghar, Mumbai \\ ${ }^{2}$ Sindhu Mahavidyalaya Panchpaoli Nagpur \\ ${ }^{3}$ V.V.P. Engineering College, Rajkot, Gujrat \\ ${ }^{4}$ Padmashree Dr. D.Y. Patil University, Navi Mumbai, 400614, India \\ ${ }^{5}$ Dr. D. Y. Patil ACS College, Pimpri, Pune \\ ${ }^{6}$ Rai foundations College CBD Belapur Navi Mumbai \\ ${ }^{7}$ Sir PP Institute of Science, Bhavnagar \\ ${ }^{8}$ Rural College of Pharmacy, D.S Road, Bevanahalli, Banglore
}

\begin{abstract}
Oncogenomics is new research sub-field of genomics, which applies high throughput technologies to characterize genes associated with cancer and synonymous with cancer genomics. The goal of oncogenomics is to identify new oncogenes or tumor suppressor genes that may provide new insights into cancer diagnosis, predicting clinical outcome of cancers, and new targets for cancer therapies. Oncoproteomics is the term used to describe the application of proteomic technologies in oncology and parallels the related field of oncogenomics. It is now contributing to the development of personalized management of cancer. Genomics has generated a wealth of data that is now being used to identify additional molecular alterations associated with cancer development. Mapping these alterations in the cancer genome is a critical first step in dissecting oncological pathways.
\end{abstract}

Keywords- Biomarkers, Drug development, Drug resistance, Gene therapy, Oncogenomics

\section{Introduction}

Oncogenomics applies high throughput technologies to characterize genes associated with cancer and is used to identify new oncogenes or tumor suppressor genes, which may provide new insights into cancer diagnosis, predicting clinical outcome of cancers and new targets for cancer therapies. The success of targeted cancer therapies such as Gleevec, Herceptin and Avastin raised the hope for oncogenomics to elucidate new targets for cancer research [1]. Oncogenomics is to allow for the development of personalized cancer treatment and focuses on the genomic, epigenomic and transcript level alterations in cancer. Comparative oncogenomics uses crossspecies comparisons to identify oncogenes and research involves studying cancer genomes, transcriptomes and proteomes in other model organisms, i.e., mice, identifying potential oncogenes, and referring back to human cancer samples to see whether homologues of these oncogenes are also important in causing cancer in humans. Comparative oncogenomics is a powerful approach to oncogene identification [2]. Mutational analysis of all gene families has been a powerful approach to oncogenomics, which has been informative. Genes from same family have similar functions, as predicted by similar coding sequences and protein domains, have been systematically sequenced in cancerous genomes to identify particular pathways which may be associated with cancer progression. Such class of families has been studied is the kinase family genes, involved in adding phosphate groups to proteins, and phosphatase family genes, involved with removing phosphate groups from proteins. [3]. The kinase and phosphatase families were first examined because of their apparent role in

transducing cellular signals of cell growth or death. In particular, more than $50 \%$ of colorectal cancers were found to carry a mutation in a kinase or phosphatase gene. Phosphatidylinositold 3-kinases (PIK3CA) gene encode for lipid kinases which were identified to commonly contain mutations in colorectal, breast, gastric, lung, and various other types of cancer $[4,5]$. The oncogenomics can be used most effectively to impact the clinical response to cancer and in advances in the clinical application of pharmacogenomics to predict response to oncology therapeutic in cancer paradigm [6]. Human cancer genomes are characterized by numerous chromosomal aberrations of uncertain pathogenetic significance. Here, in an inducible mouse model of melanoma, the characterized metastatic variants with an acquired focal chromosomal amplification that corresponds to a much larger amplification in human metastatic melanomas. Further research identified Nedd9, an adaptor protein related to p130CAS, as the only gene within the minimal common region that exhibited amplification - associated overexpression. Functional, clinical and biochemical studies established NEDD9 as a bona fide melanoma metastasis gene. NEDD9 enhanced invasion invitro and metastasis invivo of both normal and transformed melanocytes, functionally interacted with focal adhesion kinase and modulated focal contact formation, and exhibited frequent robust overexpression in human metastatic melanoma relative to primary melanoma. Comparative oncogenomics has enabled the identification and facilitated the validation of a highly relevant cancer gene 
governing metastatic potential in human melanoma [7].

\section{Oncogenomics and its application to clinical pathogenesis}

Anaplastic thyroid carcinoma (ATC) is considered one of the most aggressive malignancies, having a poor prognosis and being refractory to conventional chemotherapy and radiotherapy. Alteration in histone deacetylase (HDAC) activity has been reported in cancer, thus encouraging the development of HDAC inhibitors, whose antitumor action has been shown in both solid and hematological malignancies [8]. Genome having huge amount of information about biological functions and the interest is now directed to the study using genomic information, the genomic strategy is not clearly understood. The following 4 studies are new strategy for research: (i) models for genetic and genomic investigations of complex genetic diseases (ii) Molecular classification by global gene expression profiling (iii) Functional genomics search of disease-related genes using microarrays (iv) Acute ischemic change of mRNA expression [9]. Poly-trauma remains a medical entity with major implications, for patient's mortality, morbidity and healthcare economics. Advances in molecular medicine have improved diagnostic techniques in detecting devastating complication after major trauma. Patients at high risk of multiple organ dysfunction syndrome (MODS) or adult respiratory distress syndrome (ARDS), could be identified early, monitored and treated. Proteomics research is the systematic evaluation of proteins produced by the cell under normal or pathological circumstances. Investigating protein production will allow us to identify and modify disease natural history and treatment [10]. Early detection is essential for the control and prevention of many diseases, in cancer, which is the reason why the need for new disease markers with improved sensitivity and specificity continues to grow. Utilization of sophisticated bioinformatics tools enables the increased specificity and a relatively large quantity of high quality assays for any gene of interest. Genetic research screening is one of the fastest moving areas of medical science, particularly in oncology, and as more genes are cloned, and more disease-associated mutations discovered, the workload is set to increase considerably with the utilization of bioinformatics tools used in integration and analysis of genomic, proteomic and metabolomic profiles of cancer [11]. Genomic copy number aberrations and corresponding transcriptional deregulation in the cancer genome have been suggested to have regulatory roles in cancer development and progression. Functional evaluation of individual genes from lengthy lists of candidate genes from genomic data sets presents a significant challenge. Regional pattern recognition approaches, the most probable copy number-dependent regions and 50 potential driver genes. The gene selection process, the functional relevance of the selected genes was evaluated by estimating the prognostic significance of the selected genes. Small interference RNA mediated knockdown experiments showed proof-of-principle evidence for the potential driver roles of the genes in hepatocellular carcinoma progression (i.e., NCSTN and SCRIB). More to this, systemic prediction of drug responses implicated the association of the 50 genes with specific signaling molecules (mTOR, AMPK, and EGFR) [12]. Study on retroviral insertion mutagenesis has recently received much attention because of its adverse effects in the application of retroviral vector-based gene therapy, resulting in leukemia in certain patients. Increasing numbers of new potential oncogenes identified in retroviral screens have given a valuable basis for a better understanding of cancer related pathways in mice. Important challenges that now lie ahead are (i) to determine the relevance and causal relationship of these genes with various types of human cancer (ii) to develop strategies to identify tumor suppressor genes on a large scale, (iii) to place the disease genes into regulatory networks to better understand their role in the complex pathogenesis of cancer, and (iv) to determine their value for diagnosis refinement and therapeutic target intervention in human disease [13]. High population frequency of a germline TP53 mutation (R337H) predisposing to early cancer has led to mass newborn testing for this mutation. Newborn screening for inherited cancer research risk is complex and controversial. $\mathrm{R} 337 \mathrm{H}$ has been identified in Brazilian families with Li-Fraumeni or related syndromes predisposing to cancers in childhood (i.e., brain, renal, and adrenocortical carcinomas), adolescence (i.e., soft tissue and bone sarcomas), and young adulthood (i.e., breast cancer). Detailed assessment of the risks, benefits, and costs is needed to ensure that medical, social, and ethical justifications for newborn screening are met [14]. Caveolin-1 is the main component of caveolae membrane structures has different roles during tumorigenesis in different cancer types with varying expression profiles, indicating,w which the role of caveolin-1 varies according to tumor type. Caveolae formation and expression of Caveolin-1 (Cav-1) affect the biological consequences of such signaling pathways via particular mechanisms including receptor internalization [15-18]. 


\section{Pharmacology of oncogenomical gene therapy \\ Ras and calcineurin are members of two} independent pathways in muscle growth but their interaction is not known. Research shows that, the transfection of about $1 \%$ of the muscle fibers with dominant negative Ras (dnRas) shows a wilder effect; it stimulates the fiber growth in the entire regenerating soleus muscle, including the nontransfected fibers. Co- transfection with the calcineurin inhibitor cain/cabin prevented the growth stimulation. Injection of antibody for interleukin-4 (IL-4) also abolished the growth ameliorating effect [19]. Colorectal cancer is one of the leading causes of tumor-related morbidity and mortality worldwide. While mechanisms underlying this disease have been elucidated over the past two decades, these molecular insights have failed to translate into efficacious therapy. The oncogenomic view of cancer suggests that terminal transformation reflects the sequential corruption of signal transduction circuits regulating key homeostatic mechanisms, whose multiplicity underlies the therapeutic resistance of most tumors to interventions targeting individual pathways. The paucity of mechanistic insights into proximal pathophysiological processes that initiate and amplify oncogenic circuits preceding accumulation of mutations and transformation impedes development of effective prevention and therapy [20]. Basal-like breast cancers (BLBCs) are present serious clinical challenges as there are currently no targeted therapies available. Regulatory role of Y-box binding protein-1 (YB-1) on epidermal growth factor receptor (EGFR) overexpression in BLBC, and the therapeutic potential of inhibiting epidermal growth factor receptor (EGFR). Primary tumour tissues are evaluated for YB1 protein expression by immunostaining tissue microarrays. Ability of YB1 to regulate EGFR is evaluated using luciferase reporter, chromatin immune-precipitation (ChIP) and gel shift assays. Impact of Iressa on monolayer cell growth is measured using an ArrayScan VTI high-throughput analyser to count cell number and colony formation in soft agar is used to measure anchorage-independent growth [21]. N-myristoylation ensures the proper function and intracellular trafficking of proteins and many proteins involved in a wide variety of signaling, include cellular transformation and oncogenesis, and are myristoylated. The myristoylation of proteins is catalyzed by the ubiquitously distributed eukaryotic enzyme Nmyristoyltransferase (NMT). NMT expression is elevated in colorectal and gallbladder carcinoma. The role of HSC70 and/or enolase in the regulation of NMT leads to the development of a gene-based therapy of colorectal cancer. The interaction of oncoproteomics and oncogenomic data sets through powerful bioinformatics will yield a comprehensive database of protein properties, which will serve as an invaluable tool for cancer researchers to understand the progress of tumorigenesis [22].

\section{Immunology of oncogenomics gene therapy}

Recent research in genomics and proteomics have advance understanding of myeloma pathogenesis, recognized novel mediators of disease process, and identified new therapeutic targets which provide newer diagnostic tools for myeloma, improved monitoring of the disease status and allowed for molecular classification of the disease. Advancement in investigative techniques that have helped refine the diagnostic work up in myeloma includes use of serum free light chains, in oligosecretory myeloma, patients with renal disease and with amyloidosis; use of $\mathrm{MRI}$ and PET scan in diagnosis and managing bone disease; and use of cytogenetics and fluorescent in situ hybridization (FISH) technique to determine prognosis. The ongoing oncogenomic studies including high-throughput expression profiling, high-density single nucleotide polymorphism (SNP)-arrays and array based comparative hybridization $(\mathrm{aCGH})$ have been utilized to not only understand myeloma pathobiology, but for gene discovery, identification of biomarkers, and delineation of patient subgroups to incorporate them into therapeutic strategies and to eventually provide optimal individualized therapy [23]. Haematopoietic stem cell transplantation (HSCT) is now widely applicable to treat primary immunodeficiencies (PID). For patients with specific disorders i.e., severe combined immunodeficiency (SCID)-X1, adenosine deaminase deficiency (ADA)-SCID, X-chronic granulomatous disease (CGD) and Wiskott Aldrich syndrome (WAS), who lack a suitable human leukocyte antigen (HLA)-matched donor, gene therapy has offered an important alternative treatment option. Gene therapy can be attributed in part to the selective applications offered to gene-corrected cells, the avoidance of graftversus-host disease and to the use of preconditioning in patients with chemotherapy to facilitate engraftment of corrected cells [24]. Chronic myelogenous leukemia (CML) is associated with the Ph1 chromosome translocation, which produces a chimeric tyrosine-specific kinase gene, the product of the fusion of the BCR gene and the ABL gene. The immune system has long been implicated in the control of CML. Imatinib mesylate is currently used as the first line therapy for CP-CML patients. However, it selectively targets the ABL portion of BCR-ABL protein as a reversible tyrosine kinase inhibitor, it cannot kill the leukemic stem cells of CML. Development of such immunotherapeutic strategies would be a promising approach to treat the imatinib-treated 
CML-CP patients [25]. Oncolytic virotherapy is an emerging biotherapeutic platform for cancer treatment, which is based on selective infection and killing of cancer cells by viruses. The human respiratory syncytial virus (RSV) as an oncolytic virus, Using prostate cancer models, enhancement of RSV infectivity in vitro in the androgen-independent, highly metastatic PC-3 human prostate cancer cells compared to the non-tumorigenic RWPE-1 human prostate cells. The oncolytic efficiency of RSV was established in vivo using human prostate tumor xenografts in nude mice. The oncolytic property of RSV can potentially be exploited to develop targeted therapeutics for the clinical management of prostate tumors [26]. The utility of recombinant Sendai virus (rSeV) has been considerably examined over the last decade as a potent gene transfer candidate in a cytoplasmic gene expression system. Excessive immune responses associated with this virus administration in vivo however have limited its applicability in clinical settings as is the case with other viral vectors including adenoviruses. The mechanisms of immune responses against $\mathrm{SeV}$, ex vivo infection of immature dendritic cells (DCs) with SeV demonstrates their spontaneous maturation and activation, to create a unique, representative, and powerful agent to activate DCs, namely rSeV-modified DCs (rSeV/DCs), for use in cancer immunotherapy. Use of this system in vivo resulted in the induction of efficient antitumor immunity against vascularized rodent tumors, including melanoma, hepatocellular carcinoma, neuroblastoma, squamous cell carcinoma, and prostatic cancer. Conceptual term immunostimulatory virotherapy is used to describe the new method of cancer therapy using the $\mathrm{rSeV} / \mathrm{DCs}$ system [27]. Current research into oral cancer has revealed a large amount of information about the nature of the disease. The details of many of the genetic changes are now available, raising the possibility that they could be reversed and that the growth of the tumors could be prevented. Information has accumulated about the oral viruses that could be used as delivery systems for the new treatments. There is still no scientific evidence that oral cancer in humans can be managed by any form of genetic manipulation, or by the use of any viral vectors. Interest in the subject is maintained by promising advances in animals and by the unfortunate lack of progress in competing fields such as chemotherapy [28].

\section{Oncogenomics strategies}

Multiple myeloma (MM) remains an incurable plasma cell dyscrasia characterized by bone marrow failure, bone disease, renal disease and other complications. Clinical analysis shows extensive diversity which is based on complex cytogenetic abnormalities, and lot of interactions between myeloma cell and bone marrow stroma. Advances in understanding the biology of myeloma including the function of proteasome, molecular chaperons on the folding of intracellular proteins as well as signal transduction pathway, apoptosis-inducing cascade and oncogenomics, have brought about the development of several new therapeutic strategies [29]. Oncogenomics has identified new drug targets for genotype-specific treatments and provided strategies to validate the targets and to develop drugs. Oncogenomics research has progressed logically from molecular profiling to model systems, cancer pharmacology and clinical trials. Oncogenomics covers cutting-edge issues such as array-based diagnostics, pharmacoproteomics, pharmacogenomics and molecularly targeted therapeutics includes discussions of ethical, legal, and social issues related to cancer genomics and clinical trials [30]. KRAS mutations analysis present at a high frequency in human cancers and development of therapies targeting mutated KRAS requires cellular and animal preclinical models. Adenoassociated virus-mediated homologous recombination is inserted in the Kras G12D allele in the genome of mouse somatic cells. Levels of Kras activation in knock-in cells are comparable with those present in human cancer cells carrying the corresponding mutation. Kras-mutated cells are compared with their wild-type counterparts by gene expression profiling, leading to the definition of a mutated Kras-KI signature of 345 genes. This identification study is capable of classifying mouse and human cancers according to their KRAS mutational status, with accuracy similar to or better than published Ras signatures [31]. Retroviral insertion mutagenesis has recently received much attention because of its adverse effects in the application of retroviral vectorbased gene therapy, resulting in leukemia in certain patients. The increasing numbers of new potential oncogenes identified in retroviral screens have given a valuable basis for a better understanding of cancer. Important challenges, which now lie ahead of us are (i) to determine the relevance and causal relationship of these genes with various types of human cancer (ii) to develop strategies to identify tumor suppressor genes on a large scale, (iii) to place the disease genes into regulatory networks to better understand their role in the complex pathogenesis of cancer, and (iv) to determine their value for diagnosis refinement and therapeutic target intervention in human disease [32]. A revolution has transformed the oncology field which is characterized by two main features. The first is the introduction of the concept of individualized cancer therapy. The second is the development of drugs targeting molecules selectively altered in tumours. Tumour progression is the result of the sequential accumulation of mutations in genes 
monitoring the rates of cell birth and cell death. The molecular profiling of cancers has shown that protein and lipid kinases are frequently altered in tumour cells. 'Established' cancer cells remain somewhat 'addicted' to the deregulated activity of mutated kinases. This feature appears to be the basis for the ability of kinase inhibitors in controlling the development of a number of cancers. The therapeutic efficacy of kinase inhibitors is impaired by the emergence of tumour cells carrying 'resistance' mutations [33]. Accumulation of genomic alterations is very important process in tumor formation and progression. Comparative genomic hybridization performed on cDNA arrays i.e., cDNA aCGH, is a common method to investigate the genomic alterations on a genome-wide scale. Also, when detecting low-level DNA copy number changes this technology requires the use of noise reduction strategies due to a low signal to noise ratio [34].

\section{Oncogenomics and drug resistance}

One of the very significant accomplishments of translational oncogenomics is a realistic promise of efficient diagnostic tests that would facilitate implementation of the concept of individualized cancer therapies. Gene expression signatures (GESs) associated with the stemness state of a cell might be informative as molecular predictors of cancer therapy outcome. Stemness cancer therapy outcome predictor (CTOP) algorithm combining scores of nine stemness signatures outperforms individual signatures and demonstrates a superior prognostic accuracy in retrospective supervised analysis of large cohorts of breast, prostate, lung, and ovarian cancer patients. Stemness genomics law governs clinical behavior of human malignancies and defines epigenetic boundaries of therapy-resistant and sensitive tumors within distinct stemness/differentiation programs [35]. Technologic advances in oncogenomics, proteomics and functional genomic screens are providing mechanisms to rapidly identify the critical targets whose inactivation will lead to a substantive tumor growth arrest. Tumor tissue biomarkers, which identify those tumors most likely to respond to a specific inhibitor, are needed as a mechanism toward tailoring therapy to the individual patient with malignant glioma. Basic research is providing novel insights into the complex molecular pathways involved in the pathogenesis of malignant glioma transformation and progression [36]. Drugs, which target the insulin-like growth factor-I receptor (IGF-IR) and/or insulin receptor (IR) are currently under investigation for a variety of malignancies including breast cancer. P-IGF-IR/IR could be a predictive marker for response to IGF-IR and/or IR-targeted therapies, as these inhibitors may be of benefit in all breast cancer subtypes including those with acquired resistance to tamoxifen [37]. Pharmacogenomics is a fast-growing field of investigations that aims to further elucidate the inherited nature of inter individual differences in drug disposition and effects, with the ultimate goal of providing a stronger scientific basis for selecting the optimal drug therapy. Providing the right drug for the right patient is an important problem in the treatment of cancer, which is mainly due to the lack of information about the sensitivity of the tumor for a specific treatment modality, such as either chemotherapy or radiation treatment. Presentation highlights two approaches to identify responsiveness to treatment. Both approaches are based on the identification of expression profiles. The first approach concentrates on drug resistance and the second on the signaling pathways leading up to the death of the cell. Both approaches provide expression profiles; also, the more dynamic expression profiling as used to determine the signaling in damage cells promises to be a better determinant for the pharmacogenomic changes in expression profiles and, consequently, a potential better determinant for drug efficacy [38]. Chemoresistance is a major obstacle for successful cancer treatment. Gene amplification and altered expression are the main genetic mechanisms of tumor chemoresistance research. Limited numbers of genes were analyzed in each individual study using traditional molecular methods such as Northern and Southern blotting. TS mRNA and protein are consistently overexpressed in all drug-resistant tumor cell lines compared with the sensitive parent cell lines. Southern blot and FISH analysis demonstrated that the TS gene was amplified in 5-FU- and TDX-resistant cell lines [39]. The MAGE gene family is characterized by a conserved domain (MAGE Homology Domain) and subset of highly homologous MAGE genes (group A; MAGE-A) belong to the chromosome $X$-clustered cancer, testis antigens. MAGE-A genes are normally expressed in the human germ line and overexpressed in various tumor types; also, their biological function is largely unknown. [40]. Dietary carcinogen 2-amino-1methyl-6-phenylimidazo $(4,5-b)$ pyridine (PhIP) is a heterocyclic amine which is a common byproduct of cooked meat and fish. Although most cells undergo apoptosis when exposed to this mutagen, subsets develop resistance. Rather than die, these resistant cells persist and accumulate mutations, there by driving tumorigenesis of exposed organs within the gastrointestinal tract. By applying a highthroughput cell-based screen of 32,000 small molecules, a family of compounds that specifically inhibit the growth of PhIP-resistant cancer cells was identify. These compounds may prove useful for the treatment or prevention of 
gastrointestinal tumors arising after exposure to PhIP and related carcinogens [41].

\section{Tumours and metastases}

Grading the tumors, determining the tumor size, and axillary lymph node status, staging of the tumors are studied to extent and spread of breast carcinoma in patients and Nottingham Prognostic Index (NPI) scoring on the available material. One hundred and twenty consecutive mastectomy specimens with axillary lymph node sampling removed for breast carcinoma and received in the section of histopathology, Aga Khan University, in the year 2005, were included in the study. Standard protocols were used for the processing of the specimens, and reporting was done using a standard format incorporating all relevant tumor parameters and NPI was applied [42]. Merkel cell carcinoma (MCC) is a malignant neuroendocrine tumor of the skin that demonstrates a remarkable tendency to metastasize. However, only a few cases of MCC brain metastases have been reported in the literature. History of pituitary adenoma is a unique case of a pituitary metastasis of MCC in a 65 -year-old patient. The case is particularly novel due to the fact that the primary site of the MCC is unknown [43]. To demonstrate the unfavourable results in survival rates in patients with medial breast cancer compared to patients with laterally located tumors of the mammary gland. Treatment protocols involved breast-conserving surgery and whole-breast radiotherapy in all women, followed by a tumor bed boost dose according to risk factors for local recurrence [44]. Choriocarcinoma are commonly gestational and intrauterine or gonadal in origin. Nongestational and extragonadal disease is extremely rare with a poorer prognosis and early metastasis. The histopathologic findings of the resected tumor were that of an extensively necrotic and hemorrhagic neoplasm with combined choriocarcinomatous and adenocarcinomatous element. A strongly positive immunohistochemical staining for beta- subunit of human chorionic gonadotropin (beta-HCG) in the choriocarcinomatous component and a proper correlation with elevated serum beta-HCG levels confirmed the diagnosis [45]. Treatment methods for patients with cervical cancer of an unknown primary site (CUP) are still under discussion. The purpose of this retrospective study was to analyze the oncological follow-up of 58 patients treated for cervical CUP [46]. To determine the presence of disseminated tumor cells in bone marrow or apheresis product, and also to evaluate the clinical significance of contaminated products and the efficacy of CD34 (+) selection and high-dose chemotherapy in patients with Stage III breast cancer [47]. Proteins of the lysyl oxidase (LOX) family are important modulators of the extracellular matrix. They have an important role in the tumour development as well as in tumour progression. To evaluate the diagnostic and prognostic value of the LOX protein in oral and oropharyngeal squamous cell carcinoma (OSCC) we performed QRT-PCR and immunohistochemical analysis on two tissue microarrays. In a multivariate analysis, high LOX expression was an independent prognostic factor, predicting unfavourable overall survival. LOX expression is an independent prognostic biomarker and a predictor of lymph node metastasis in OSCC. LOX overexpression may be an early phenomenon in the pathogenesis of OSCC and thus an attractive novel target for chemopreventive and therapeutic strategies [48].

\section{Oncogenomics and clinical mechanism}

Whole genome expression profiling of 28 patient prostate tumor samples and 12 normal prostate samples which was used to identified 55 upregulated and 60 downregulated genes significantly changed in prostate tumor samples compared to normal prostate tissues. Expression profiling of HOXC6 siRNA transfections and HOXC6 overexpression identified neutral endopeptidase (NEP) and insulin-like growth factor binding protein-3 (IGFBP-3) as potential proapoptotic repression targets of HOXC6. HOXC6 may be a novel potential therapeutic target for prostate cancer [49]. Chromosomal translocation is the best-characterized genetic mechanism for oncogene activation. Cancer lines representing diverse tissue origins identified genes residing in amplification hotspots and discovered an unexpected frequency of genes activated by molecular mechanism. Integrating gene dosage with expression data validated the downstream impact of the novel amplification events in both cell lines and clinical samples. For example, multiple downstream components of the EGFR-family-signaling pathway, including CDK5, AKT1 and SHC1, are overexpressed as a direct result of gene amplification in lung cancer [50]. Using FISH-based techniques, rearrangements of the immunoglobulin heavychain $(\mathrm{IgH})$ locus at $14 \mathrm{q} 32$ have been found in the majority of cases of multiple myeloma (MM). Some of these $\mathrm{IgH}$ translocations are recurrent and we have characterized the genomic breakpoints of seven $t(4 ; 14)$ translocations from MM patients, using a combination of vectorette and conventional polymerase chain reaction methods, the aim being to understand the molecular mechanism leading to MM. The chromosome 14q32 breakpoints in these reciprocal translocations are believed to be located in the $\mathrm{IgH}$ switch (S) region and a further downstream $S$ region with deletion of intervening DNA occurring as a result of aberrant class switch recombination (CSR); this was seen in five of the cases analysed [51]. Histone deacetylase inhibitors (HDI) are a promising new approach to 
the treatment of cancer. HDIs have been shown to induce differentiation, cell cycle arrest, and apoptosis in a variety of transformed cell lines; inhibit tumor growth in animal models; and show antitumor activity in clinical trials. Understanding of the molecular determinants of resistance to HDIs may provide the basis for therapeutic combinations with improved clinical efficacy. Poor response to treatment could be linked to systemic factors like pharmacokinetics or to tumor-specific factors both at the level of the malignant cells (tumor intrinsic) or the tumor microenvironment [52]. Deleted in liver cancer (DLC-1) gene is frequently deleted in hepatocellular carcinoma. However, little is known about the genetic status and the expression of this gene in gastric cancer. Northern and Southern analysis showed that seven of nine human gastric cancer cell lines did not express DLC-1 mRNA, but contained the DLC-1 gene. Primary gastric cancers were also shown to be methylated; demonstrating that methylation of the DLC-1 CpG island is not uncommon in gastric cancer [53].

\section{Drug resistance mechanism}

Melanoma is the most aggressive form of skin cancer. Patients with advanced disease, such as lymph node involvement and distant metastases, have 5-year survival rates of $50 \%$ and $10-20 \%$, respectively. This poor prognosis largely results from resistance to conventional chemotherapy, namely cytotoxic drugs. The basis for drug resistance in melanoma is most likely dysregulation of apoptosis, although other mechanisms including drug transport, detoxification, and enhanced DNA repair may also play a role. Defects at multiple levels and in both major apoptotic pathways have been described in melanoma. An inhibitor of apoptosis, termed survivin, that is expressed in melanoma and required for maintenance of melanoma cell viability. Targeting of survivin and other apoptotic regulators increases the sensitivity of melanoma cells to cytotoxic drugs, and may provide a promising new therapeutic approach to cancer [54]. Clinical application of platinum compounds in cancer chemotherapy has prompted a search for new platinum agents. To search for platinum agents with novel profiles of activity, clustered image maps, the COMPARE algorithm, and other numerical methods to analyze platinum compounds and anticancer drug screen which is tested against the screen's 60 diverse human cancer cell lines (the $\mathrm{NCl}-60$ ). Many of the compounds retained activity against the resistant cells, which provide evidence that they differ from cisplatin and oxaliplatin, not only in their selective activity against the various $\mathrm{NCl}-60$ cell types, but are also in their susceptibility to mechanisms of resistance. Biologically interpretable molecular predictor of activity is consistent with the idea that platinum compounds have multiple intracellular targets and that cells can have multiple mechanisms of resistance [55]. P-glycoprotein is a transmembrane protein thought to function as an efflux pump to detoxify cells. It is associated with multidrug resistance in laboratory systems and has recently been found in human tumors associated with in vitro and clinical drug resistance. The dose-limiting toxicity of the verapamil infusion is temporary cardiac dysfunction including hypotension, congestive heart failure, and cardiac arrhythmia [56]. The Bcell neoplasms, non-Hodgkin's lymphoma and multiple myeloma frequently become drug resistant, despite initial responses to chemotherapeutic drugs. Tumor cells from patients with clinically drug-refractory disease are evaluated by immuno-histochemical staining for monoclonal immunoglobulin (lg) expression, nuclear proliferation antigen, $\mathrm{P}$-glycoprotein $(\mathrm{P}$ gly) expression and other cellular antigens. P-gly is detected on tumor cells from six of eight patients with drug-resistant disease. Verapamil increased the intracellular accumulation of doxorubicin and vincristine in vitro for both a $\mathrm{P}$ gly-positive myeloma cell line and tumor cells from two patients with end-stage myeloma, that over- expressed P-gly. The dose-limiting side effect associated with the addition of verapamil to chemotherapy is temporary impairment of cardiac function, manifest as hypotension and cardiac arrhythmia [57]. Selection for in vitro drug resistance can result in a complex phenotype with more than one mechanism of resistance emerging concurrently or sequentially. Examination of emerging mechanisms of drug resistance during selection with mitoxantrone in the human myeloma cell line 8226. Increased resistance to mitoxantrone may result from reduced intracellular drug accumulation, altered nuclear/cytoplasmic drug distribution, and alterations in topoisomerase II activity [58]. Doxorubicin-selected lung cancer cell line H69AR is resistant to many chemotherapeutic agents. Also, like most tumor samples from individuals with this disease, it does not overexpress $P$ glycoprotein, a transmembrane transport protein that is dependent on adenosine triphosphate (ATP) and is associated with multidrug resistance. Complementary DNA (cDNA) clones corresponding to messenger RNAs (mRNAs) overexpressed in H69AR cells were isolated and hybridized to an mRNA of 7.8 to 8.2 kilobases that was 100 - to 200 -fold more expressed in H69AR cells relative to drug-sensitive parental H69 cells. Reversion to drug sensitivity was associated with loss of gene amplification and a marked decrease in mRNA expression. The mRNA encodes a member of the ATP-binding cassette transmembrane transporter superfamily [59]. Integrin-mediated adhesion influences cell survival and may prevent programmed cell death. Little is known about how drug-sensitive tumor 
cell lines survive initial exposures to cytotoxic drugs and eventually select for drug-resistant populations. Factors that allow for cell survival following acute cytotoxic drug exposure may differ from drug resistance mechanisms selected for by chronic drug exposure. This cell adhesion mediated drug resistance, or CAM-DR, was not due to reduced drug accumulation or upregulation of anti-apoptotic Bcl-2 family members. Flow cytometry determine, myeloma cell lines selected for drug resistance, with doxorubicin or melphalan, overexpress VLA-4. Functional assays revealed a significant increase in 4-mediated cell adhesion in both drug-resistant variants compared with the drug-sensitive parent line [60].

\section{Drug oncogenomics}

Basal-like breast cancers (BLBC) frequently overexpress the epidermal growth factor receptor (EGFR) and subsequently have high levels of signaling through the MAP kinase pathway, which is thought to contribute to their aggressive behavior. Using Motif scan we identified p90 ribosomal S6 kinase (RSK) as a potential candidate for activating YB-1 [61]. Gene expression analysis study is used to subtype breast cancers such as the most aggressive tumors are identified, but translating this into clinical practice can be cumbersome. The goal is to develop a universal biomarker, which distinguishes patients at high risk across all breast cancer subtypes. Y-box binding protein-1 (YB-1), a transcription/translation factor, was a marker of poor prognosis in a cohort of 490 patients with breast cancer, but the study was not large enough to subtype the cancers was reported. To investigated whether YB-1 identifies patients at risk for either reduced relapse free survival or decreased breast cancer specific survival (BCSS) across all tumor subtypes by evaluating 4,049 cases [62]. Multiple myeloma is a plasma cell malignancy characterized by complex heterogeneous cytogenetic abnormalities and bone marrow microenvironment promotes multiple myeloma cell growth and resistance to conventional therapies. Although multiple myeloma remains incurable, novel targeted agents, used alone or in combination, have shown great promise to overcome conventional drug resistance and improve patient outcome. Recent oncogenomic research have further advance understanding of the molecular pathogenesis of multiple myeloma, providing the framework for new prognostic classification and identifying new therapeutic targets [63]. The epidermal growth factor receptor (EGFR) is integral to basal-like and human epidermal growth factor receptor-2 (Her-2)overexpressing breast cancers which is associated with poor prognosis, the majority of which express high levels of EGFR. MDA-MB-
468 and SUM 149 are basal-like breast cancer (BLBC) cells that were used for study because they express high levels of activated PDK-1, YB1, and EGFR compared with the immortalized breast epithelial cell line 184htrt. [64]. DNA chip technology has led to an explosion of oncogenomic analyses and generating a wealth of data and uncovering the complex gene expression patterns of cancer. Due to the lack of a unifying bioinformatics resource, the majority of these data sit stagnant and disjointed following publication, massively underutilized by the cancer research community. ONCOMINE is a cancer microarray database, which is web-based datamining platform aimed at facilitating discovery from genome-wide expression analyses. Differential expression analyses comparing most major types of cancer with respective normal tissues as well as a variety of cancer subtypes and clinical-based and pathology-based analyses are available for exploration. Gene sets can be limited to clinically important annotations including secreted, kinase, membrane, and known gene-drug target pairs to facilitate the discovery of novel biomarkers and therapeutic targets [65].

\section{Translational and functional oncogenomics}

To classify the multiplicity of genetic abnormalities in five repertoires that is rapidly assessable and useful for stratification in clinical trials: allelic imbalance, aberrant promoter methylation, gene mRNA overexpression, microtubule alterations, and polymorphisms. Allelic imbalance refers to chromosomal instability, which is a major feature of cancer, and innovative techniques used in colorectal cancer should also be implemented in lung cancer. Methylation techniques have shown that these epigenetic changes commonly occur at the same frequency in numerous genes, both well-known (FHIT, APC, p16) and recently discovered (TMS1, RASSF1) in non-small cell lung cancer and in breast cancer. Innovative techniques like quantitative polymerase chain reaction can determine gene expression profiles, mainly overexpression of mRNAs, which may be related to resistance to specific cytotoxic drugs [66]. Complementary competences and resources have been brought together in the TRANSFOG Consortium to reach the following integrated research objectives: (i) execution of canceroriented genomic screenings on tumor tissues and experimental models and merging of the results to generate a prioritized panel of candidate genes involved in cancer progression and metastasis; (ii) setup of systems for highthroughput delivery of full-length cDNA, for gainof-function analysis of the prioritized candidate genes; (iii) collection of vectors and oligonucleotide for systematic, RNA interferencemediated down-regulation of the candidate 
genes; (iv) adaptation of existing cell-based and model organism assays to a systematic analysis of gain and loss of function of the candidate genes, for identification and preliminary validation of novel potential therapeutic targets; (v) proteomic analysis of signal transduction and protein-protein interaction for better dissection of aberrant cancer signaling pathways; (vi) validation of the diagnostic potential of the identified cancer genes towards the clinical use of diagnostic molecular signatures; (vii) generation of a shared informatics platform for data handling and gene functional annotation [67]. Explosion of information generated by largescale functional genomics technologies has resulted in an exponential increase in the number of potential genes and proteins available for pharmaceutical and diagnostic research development. The primary challenge is to develop a strategy to effectively integrate and extract meaning from the human genomic sequence information that has been generated since the start of the Human Genome Project [68]. Long terminal repeats (LTRs) of human endogenous retroviruses (HERVs) have been reported to serve as alternative promoters in functional genes. The GSDML (gasdermin-like protein) gene located on human chromosome 17q21 has been found to be an oncogenomic recombination hotspot. The transcripts of this LTR-derived promoter were widely distributed in various human tissues and cancer cells, whereas the transcripts of the cellular promoter were found only in stomach tissues and some cancer cells [69]. The increasing number of proteomic and DNA-microarray studies is continually providing a steady acquisition of data on the molecular abnormalities associated with human tumors. Rapid translation of the accumulating biological information into better diagnostics and more effective cancer therapeutics in the clinic depends on the use of robust function-testing strategies. The strategies should allow identification of molecular lesions that are essential for the maintenance of the transformed phenotype and enable validation of potential drug-targets. The tetracycline regulated gene expression/suppression systems (Tetracyclinesystems) developed and optimized by bioengineers over recent years seem to be very well suited for the function-testing purposes in cancer research [70]. Serial analysis of gene expression (SAGE) provides quantitative and comprehensive expression profiling in a given cell population. To define gene expression alterations in Barrett's-related adenocarcinoma (BA), eight SAGE libraries were produce and obtained a total of 457,894 expressed tags with $32,035(6.9 \%)$ accounting for singleton tags [71]. The overexpression of the epidermal growth factor receptor (EGFR) and HER-2 underpin the growth of aggressive breast cancer; still, it is unclear what governs the regulation of these receptors. Recently determined that the Y-box binding protein-1 (YB-1), an oncogenic transcription/translation factor, induced breast tumor cell growth in monolayer and in soft agar. Knocking down YB-1 with small interfering RNA suppressed the expression of EGFR and HER-2. This was coupled with a decrease in tumor cell growth [72]. Thyroid cancer is frequently associated with the oncogenic conversion of the RET receptor tyrosine kinase. RET gene rearrangements, which lead to the generation of chimeric RET/papillary thyroid carcinoma (PTC) oncogenes, occur in PTC, whereas RET point mutations occur in familial multiple endocrine neoplasia type 2 (MEN2) and sporadic medullary thyroid carcinomas (MTC). The findings identify RET as a novel substrate of PTPRJ and suggest that PTPRJ expression levels may affect tumor phenotype associated with RET/PTC1 and RET (C634R) mutants and resistance to PTPRJ may be part of the mechanism of RET oncogenic conversion secondary to the M918T mutation [73]. Development of techniques for oncogenomic analyses, such as array comparative genomic hybridization, messenger RNA expression arrays and mutational screens have come to the fore in modern cancer research. Studies utilizing these techniques are able to highlight panels of genes that are altered in cancer. The candidate cancer genes must then be scrutinized to reveal whether they contribute to oncogenesis or are coincidental and noncausative. Computational methods for the prioritization of candidate are (i) proto-oncogenes and (ii) tumour suppressor genes from oncogenomic experiments. Computational classifiers use different combinations of sequence and functional data including sequence conservation, protein domains and interactions, and regulatory data which are able to distinguish between known cancer genes and other human genes. Computational cancer gene classification as a useful method of prioritizing candidate cancer genes identified in oncogenomic studies [74]. Identification of molecular pathways that are deregulated in cancer will not only elucidate underlying tumorigenic mechanisms, but may also help to determine the classes of drugs that are used for treatment. In kidney cancer, a spectrum of histological subtypes exists that are characterized both by distinct molecular signatures and increasingly by distinct molecular pathways that are deregulated in each subtype. For example, the VHL/hypoxia pathway is wellknown to be deregulated in clear cell renal cell carcinoma (RCC) whereas in papillary RCC activation of the HGF/Met pathway has been implicated. Gene expression data is used to identify both cytogenetic abnormalities and molecular pathways that are deregulated in RCC. Predicting pathway abnormalities can be linked to 
predicted cytogenetic abnormalities to identify likely candidate genes. RCC have the potential to open up new avenues of molecular research and may have significant diagnostic and therapeutic implications [75]. The OncoDB.HCC (http://oncodb.hcc.ibms.sinica.edu.tw) is based on physical maps of rodent and human genomes containing quantitative trait loci of rodent HCC models and various human HCC somatic aberrations including chromosomal data from loss of heterozygosity and comparative genome hybridization analyses, altered expression of genes from microarray and proteomic studies, and finally experimental data of published HCC genes. Comprehensive integration of HCC genomic aberration data avoids potential pitfalls of data inconsistency from single genomic research approach. It provides lines of evidence to reveal somatic aberrations from levels of DNA, RNA to protein. OncoDB, HCC graphically presents comprehensive $\mathrm{HCC}$ data integration, reveals important HCC genes and loci for positional cloning and functional studies, and discloses potential molecular targets for improving HCC diagnosis and therapy [76].

\section{Therapeutic and experimental strategy}

Co-injection of conventional single-stranded adeno-associated virus 2 (ssAAV2) vectors containing the enhanced green fluorescent protein (EGFP) gene with self-complementary (sc) AAV2-T cell protein tyrosine phosphatase (TC-PTP) and scAAV2-protein phosphatase 5 (PP5) vectors resulted in a 16-fold increase in EGFP expression in primary murine hepatocytes in vivo. The strategy was further optimized to achieve transgene expression at reduced vector/helper-virus doses which included the use of scAAV helper-viruses containing (i) hepatocyte-specific promoters, (ii) tyrosinemutant AAV2 capsids, and (iii) additional AAV serotype vectors known to efficiently transduce hepatocytes. No adverse effect on hepatocytes was observed under any of the experimental conditions given [77]. The goal of the study was to investigate the therapeutic potential of a novel immunotherapy strategy resulting in immunity to localize or metastatic human papillomavirus 16transformed murine tumors. Animals bearing E7expressing tumors were coimmunized by lymph node injection with E7 49-57 antigen and TLR3ligand (synthetic dsRNA). In situ cytotoxicity assays and identification of tumor-infiltrating lymphocytes and $T$ regulatory cells were used to assess the mechanisms of treatment resistance in bulky disease. Chemotherapy with cyclophosphamide was explored to augment immunotherapy in late-stage disease [78]. Sepsis is exceedingly burdensome for hospital intensive care unit caregivers and its incidence, as well as sepsis-related deaths, is increasing steadily. Sepsis is characterized by a robust increase in
NO production throughout the organism that is driven by the inducible NOS (iNOS). However, nitric oxide (NO) is an important factor in the development of septic shock and is synthesized by nitric oxide synthase (NOS), an enzyme expressed by a variety of cells including vascular endothelium, macrophage and neutrophils. The effects of $\mathrm{NO}$ on leukocyte functions, and the underlying mechanisms, are relatively unknown. $\mathrm{NO}$ induces post-translational modifications of key proteins in targeted processes with the potential of deterring cellular physiology. The manipulation of NO distribution in septic patients, used in conjunction with conventional treatments aimed at restoring normal immune functions, may represent a valuable therapeutic strategy [79]. Malignant melanoma is a highly aggressive, drug-resistant cancer and virotherapy is a novel therapeutic strategy based on cancer cell lysis through selective virus replication. The growth compromised herpes simplex virus type 2 (HSV2) mutants, DeltaPK, has strong oncolytic activity for melanoma largely caused by a mechanism other than replication-induced cell lysis. DeltaPK is a promising virotherapy for melanoma that functions through virus-induced programmed cell death pathways [80]. Hepatocellular carcinoma (HCC), one of the most lethal cancers, results in more than one million fatalities worldwide every year. Resveratrol, a naturally occurring antioxidant and antiinflammatory agent found in grapes and red wines, inhibits carcinogenesis with a pleiotropic mode of action. One of the mechanisms of resveratrol-mediated chemoprevention of hepatocarcinogenesis could be related to its antiinflammatory action through hepatic cyclooxygenase (COX-2) inhibition. The objective of the study was to evaluate the role of resveratrol on cardiac performance during experimental hepatocarcinogenesis initiated with diethylnitrosamine and promoted by phenobarbital. The cardiotoxicity of resveratrol was assessed by monitoring the cardiac function using transthoracic echocardiography as well as Western blot analysis of cardiac tissue. The chemopreventive effects of resveratrol reflect in histopathological assessment of hepatic tissues. Resveratrol did not exhibit any cardiotoxicity but rather improved the cardiac function in a doseresponsive fashion [81]. Lipopolysaccharide (LPS) has been recognized as a major player in the pathogenesis of sepsis and neutralization of LPS or inhibition of its signal transduction mechanism is promising new treatment strategy in preclinical experiments which is unsuccessful in clinical trials. LPS activates Toll-like receptor 4 (TLR4) and induces pro-inflammatory and antiinflammatory responses, the altered innate and adaptive immune responses eventually lead to the immunosuppressive state. The future therapeutic efforts in sepsis should focus on the immunosuppressive state. The data on 
therapeutic strategies targeting LPS, TLR4 and single cytokine in sepsis and discuss the experimental and clinical evaluation of the immunomodulatory action of glycine and berberine. Berberine in combination with yohimbine can modulate host immune responses in endotoxemia; it seems worthwhile to conduct clinical trials on the safe and efficacy of this new immunomodulatory therapy [82]. Malignant tumors usually involve a relatively hypoxic state, which induces overexpression of hypoxiainducible factor-1alpha (HIF-1alpha) to satisfactorily enable the tumor to survive whose inhibition of the mammalian target of rapamycin (mTOR) pathway including HIF-1alpha is expected to play a major role in suppression of tumor cell growth, having recently drawn much attention as an anti-cancer therapeutic strategy for various malignant tumors [83].

\section{Oncogenomics and biomarker in drug development}

Oncoproteomics is also used to describe the application of proteomic technologies in oncology and parallels the related field of oncogenomics which contribute to the development of personalized management of cancer. Proteomic technologies are used for the identification of biomarkers in cancer, which facilitate the integration of diagnosis and therapy of cancer. Molecular diagnostics, laser capture microdissection and protein biochips are among the technologies that are having an important impact on oncoproteomics. Continued refinement of techniques and methods to determine the abundance and status of proteins in vivo holds great promise for the future study of normal cells and the pathology of associated neoplasms. Personalized cancer therapy is expected to be in the clinic by the end of the first decade of the 21 st century [84]. There are a number of causes of traumatic brain injury (TBI), the armed conflict in Iraq and Afghanistan has brought this disorder to the attention of the global community. A biomarker that would enable army medics to rapidly diagnose the severity of $\mathrm{TBI}$ on the battlefield would be a huge asset. As the study of TBI has not historically attracted the proteomic research community's interest as other disorders have, such as cancer. On the positive side many of the analytical and technological challenges that were overcome in the development of biofluid proteomic methods are now being applied to the study of TBI [85]. Semiconductor quantum dots are nanoparticles with intense, stable fluorescence, and could enable the detection of tens to hundreds of cancer biomarkers in blood assays, on cancer tissue biopsies, or as contrast agents for medical imaging. With the emergence of gene and protein profiling and microarray technology, highthroughput screening of biomarkers has generated databases of genomic and expression data for certain cancer types, and has identified new cancer-specific markers. Quantum dots have the potential to expand this in vitro analysis, and extend it to cellular, tissue and whole-body multiplexed cancer biomarker imaging [86]. Proteomic technologies are used for the identification of biomarkers in cancer, which will facilitate the integration of diagnosis and therapy of cancer. The discovery of protein patterns developed by the US Food and Drug Administration/National Cancer Institute Clinical Proteomics Program is capable of distinguishing cancer and disease-free states with high sensitivity and specificity and will also facilitate the development of personalized therapy of cancer. Examples of application are given for breast and prostate cancer and a selection of companies and their collaborations that are developing application of proteomics to personalized treatment of cancer. Personalized cancer therapy is expected to be in the clinic by the end of the first decade of the 21st century [87]. The genetic biomarkers increasingly which is gaining recognition in the $\mathrm{N}$-acetyltransferase 2 (NAT2) gene. Individuals with the slow metabolism variant of NAT2 are thought to be at higher risk of developing bladder cancer [88]. Biomarkers are emerging that can indicate whether individuals might be predisposed to develop cancer genes at sometime in their lives. Well-publicised examples of genetic biomarkers are the breast cancer genes BRCA1 and BARC2. Young women can be screened for these genes, but even if they are carriers it is not entirely certain that they will develop breast cancer [89]. Removal of both healthy breasts as a preservative measure in response to a positive BRCA assay is still regarded by many as too drastic a measure, although some women do take this step. Either way, the patients testing positive carry a large psychological toll, living in the knowledge that they are at high risk of developing cancer [90]. Expression genomics can be defined as the study of the dynamic transcriptomes and its regulatory elements. Various technologies are available, which can assess transcripts on a genome-wide scale over time and across many samples. New comprehensive and dynamic database is being used to decipher signalling pathways and to identify new biomarkers and targets. Biomarkers emerging from these research studies have prognostic potential and can be used to predict therapeutic outcome. The multiplex nature of this approach not only telescopes the time to discovery, but also allows for detection of complex interactions. Taken together, these capabilities, if carefully used, can speed drug development, enhance the identification of potent drug combinations and identify patient 
populations that will benefit from these new drugs [91].

\section{Delivery of cancer biologics \\ Penetration \& distribution}

Human monoclonal antibodies are promising agents for the development of more selective anticancer therapeutics. The tumor-targeting efficiency of most anticancer antibodies is severely limited by their poor penetration into the tumor mass. Peptide derived from the HIV TAT protein could improve the distribution of cytoplasmic reporter proteins when administered systemically as fusion proteins or cross-linked chimeras. Conjugation to TAT peptides could improve the tumor targeting properties of scFv (L19)-Cys: an engineered human antibody fragment specific for the ED-B domain of fibronectin, a marker located in the modified extracellular matrix surrounding tumor neovasculature. TAT peptides, consisting either of L-amino acids or D-amino acids, can efficiently transduce target cells when conjugated to fluorophores and/or antibody fragments, suggesting a receptor-independent cell entry mechanism. TAT peptides composed of D-amino acids may significantly reduce proteolytic degradation while the poor biodistribution properties of antibody-TAT conjugates cast doubts over the applicability of this methodology for the delivery of biopharmaceuticals in vivo [92]. Controlled release delivery of carmustine from biodegradable polymer wafers was approved as an adjunct to surgical resection in the treatment of recurrent glioblastoma multiforme after it was shown in clinical trials to be well tolerated and effective. Drug distribution and clearance have been extensively studied in both rodent and nonhuman primate brains at various times after implantation. To characterise the degradation of the polymer matrix, the release kinetics of carmustine and the metabolic fate of the drug and polymer degradation products have been conducted both in vitro and in vivo. Metabolic elimination studies of the polymer degradation products have demonstrated that sebacic acid monomers are excreted from the body in the form of expired $\mathrm{CO}(2)$, whereas 1,3-bis-(pcarboxyphenoxy) propane monomers are excreted primarily through the urine. The presence of significant convective flows due to postsurgical oedema may augment the diffusive transport of drug in the hours immediately after wafer implantation, leading to a larger short-term spread of drug [93].

\section{Selective targeting of tumors}

Lipiodol, an oily contrast medium, is utilized to deliver the anticancer agent SMANCS to the target tumor in which the tumor selective delivery of 2,500 fold more than plasma was confirmed with prolonged retention in the tumor tissue whose tumor targeting is accomplished by the arterial injection of the oily formulation of the drug. Method utilizes unique vascular properties of tumor tissue. SMANCS is a derivative of neocarzinostatin conjugated with copolymer of styrene and maleic acid. It has much propronounced lipophilicity, stability against various harsh environments and exerts a potent cytotoxicity. Therapeutic effect of the drug to unresectable primary hepatoma is much better than the conventional method [94]. Arterially administering an oily anticancer agent was successfully established for the selective targeting of metastatic lymph nodes. A high molecular weight anticancer agent, a conjugate of copolymer (styrene maleic acid) to neocarzinostatin (SMANCS) was prepared and dissolved in a lymphographic oily contrast medium, Lipiodol (SMANCS/Lipiodol). SMANCS/Lipiodol was administered intraoperatively to eight patients with colorectal cancer and preoperatively to one patient with gastric cancer with lymph node metastases [95]. Treatment of solid tumor, in particular, primary and metastatic liver cancer using a hydrophobicpolymer conjugated macromolecular anticancer agent, smancs is described. Smancs was dissolving in a lipid contrast medium, Lipiodol, as an injection into the feeding artery. The marked antitumor effect was observed in both experimental animals and human trials. The selective remaining of Lipiodol in the tumor for long period may help follow-up study as well [96]. Nonsteroidal anti-inflammatory drugs (NSAIDs) are found to be potential chemopreventive agents of colorectal cancer. Celecoxib, an NSAID with selective cyclooxygenase-2 inhibition, was proved to be effective for the prevention of colon cancer in patients with familial adenomatous polyposis (FAP) and sporadic polyps. The amount of celecoxib released from the matrix tablets at different time intervals was estimated by a HPLC method. Guar gum matrix tablets released only $2-4 \%$ of celecoxib in the physiological environment of stomach and small intestine depending on the proportion of guar gum used in the formulation [97]. Use of monoclonal antibodies for immunotherapy has been validated by the commercialization of multiple monoclonal antibody products for oncology, infectious diseases and autoimmune diseases. In addition to their application as 'naked' antibodies, they have been used as delivery vehicles for cytotoxic agents to cancer cells. The exquisite specificity of antibodies can also be exploited to initiate and/or enhance the immune response to tumors or infectious agents by targeting the relevant antigen to antigenpresenting cells (APCs). Antibody-targeted vaccines (ATVs) have demonstrated remarkable activity in preclinical models by eliminating the need for adjuvant and repetitive boosting, 
overcoming immunological non-responsiveness, inducing mucosal immunity and eliciting therapeutic cytotoxic T-cell-mediated antitumor effects. Selective targeting to appropriate receptors on APC subsets combined with strong activating signals is important for generating potent cytolytic T-cell responses [98]. The significant advances in conventional therapeutic approaches to colorectal cancer (CRC), most patients ultimately die of their disease. Dissecting the molecular mechanisms underlying CRC progression will not only accelerate the development of novel cancer-selective drugs but will also enable the therapeutic regimen to be personalized according to the molecular features of individual patients and tumors. The novel insights into $\mathrm{CRC}$ biology that are paving the way to the development of molecular therapies and summarize the results from recent clinical trials demonstrating that agents targeting tumorspecific molecular derangements can significantly improve the therapeutic efficacy of conventional chemotherapy [99]. Replication-competent vectors are derived from attenuated viruses whose genes, that are nonessential for replication in cultured cells in vitro, are either mutated or deleted. The removal of one or more nonessential genes may reduce pathogenicity without requiring a cell line to complement growth. Herpes simplex viruses (HSV) are potential vectors for several applications in human healthcare. These include delivery and expression of human genes to cells of the nervous systems, selective destruction of cancer cells, prophylaxis against infection with HSV or other infectious diseases, and targeted infection to specific tissues or organs [100]. Systemic application of nucleic acid drugs requires delivery systems that overcome the poor pharmacokinetics, limited biodistribution, and inefficient uptake of nucleic acids. PEGylated liposomes show considerable promise because of their intrinsic ability to accumulate at disease sites and facilitate transfection of target cells. To achieve high levels of selective gene expression at distal tumor sites a PEGylated liposome for the systemic administration of plasmid DNA was used. This phenomenon is characterized by a loss of disease site targeting, accelerated clearance from the blood, and acute hypersensitivity whose effects was fully attributable to a surprisingly robust, long-lived antibody response generated against polyethylene glycol (PEG), which results from the strong adjuvant effect of the plasmid payload. Immunogenicity may be substantially reduced by modifying the alkyl chain of the PEG-lipid conjugate, thereby allowing successful repeat dosing of the modified plasmid formulations without adverse side effects [101]. Recent progress in molecular biology introduced a concept of molecular targeting therapy against many diseases including neurosurgical disorders. Molecular targeting therapy is promising, because targeting a disease-specific molecule it may provide cure without inducing unfavourable side effects which is focusing mainly on CNS (central nervous system) malignancies, the current development of molecular targeting therapy with a special concern to strategies using DNA, RNA, immunotoxin, and molecular selective inhibitors. Strategies using DNA include gene therapy and DNA vaccine. RNA interference (RNAi) is one of the promising molecular targeting strategies using RNA. Immunotoxins such as recombinant chimeric cytotoxin consisting of interleukin-13 and a truncated exotoxin produced by the Pseudomonas aeruginosa bacterium is now under clinical investigation. Visualization of drug delivery, while achieving effective drug distribution using convection-enhanced delivery (CED), may also contribute to the disease targeting. These strategies together should help us develop the effective 'disease-targeting therapy' for CNS disorders [102]. Tumorassociated carbohydrate antigens and glycopeptide antigens derived from, for example, the MUC1 mucin glycoprotein, are attractive targets for the immunotherapy of cancer, owing to their expression by malignant cells. The use of synthetic glycopeptides as partial or full components of vaccines targeting these antigens is a favorable approach for the development of cancer immunotherapies because of their ability to be generated as homogenous formulations. Tremendous progress has been made in identifying the structural components of a synthetic vaccine required to elicit a highly selective and strong immune response, as well as appropriate carriers for their multivalent presentation. It is anticipated that advances in organic synthesis will ultimately aid the development of a fully synthetic vaccine incorporating important glycopeptide antigens for the treatment of cancer [103]. Single-walled carbon nanotubes (CNT) are mechanically robust graphene cylinders with a high aspect ratio that are comprised of $\mathrm{sp}(2)$-bonded carbon atoms and possessing highly regular structures with defined periodicity. Single-walled carbon nanotubes exhibit unique mechanochemical properties, which can be exploited for the development of new novel drug delivery platforms. Hypothesized that novel prototype nanostructures consisting of biologics, radionuclides, fluorochromes, and Single-walled carbon nanotubes could be synthesized and designed to target tumor cells [104]. There are extensive DNA changes in tumor cells and the genes of tumor cells continuously mutate at a high rate. While this can provide therapeutic targets, it makes it unlikely that an agent that is selective for a single target will work against all cells in a tumor. However, it may be 
possible to use tumor epitopes as sentinels to engage adaptive and innate immunological mechanisms and create a tumor destructive environment effective also against variant cells that have lost a given antigen or their ability to present it. Therapeutic tumor vaccines, in combination with the targeting, to tumors, of costimulatory molecules such as antiCD137scFv, or lymphokines such as GMCSF, will expand anti-tumor responses for therapeutic benefit when used as an adjunct to surgery and chemotherapy [105].

\section{Engineering for delivery}

Colonic drug delivery has gained increased importance not just for the delivery of the drugs for the treatment of local diseases associated with the colon but also for its potential for the delivery of proteins and therapeutic peptides. For successful colonic delivery, a drug needs to be protected from absorption and /or the environment of the upper gastrointestinal tract (GIT) and then be abruptly released into the proximal colon, which is considered the optimum site for colon-targeted delivery of drugs. Colon targeting is naturally of value for the topical treatment of diseases of colon such as Chron's diseases, ulcerative colitis, colorectal cancer and amebiasis. Peptides, proteins, oligonucleotides and vaccines pose potential candidature for colon targeted drug delivery [106]. Purpose of our efforts is to trigger the immune destruction of established cancer. Interleukin (IL)-2 can mediate the regression of tumors in patients with melanoma and renal cell carcinoma. In animal models, the antitumor effects of $\mathrm{IL}-2$ are mediated by $T$ lymphocytes. Stimulation with specific antigen can enhance the ability of $T$ cells to respond to IL-2 by triggering the rapid upregulation of the high-affinity IL-2 receptor. To design recombinant and synthetic vaccines capable of preferentially priming $\mathrm{T}$ cells with specificity for tumor cells [107]. The cytokines interleukin (IL)-12 and IL-18 exert a variety of immune-regulatory functions including interferon (IFN)-gamma production and cytotoxic T lymphocyte (CTL) and natural killer (NK) activation. Investigation was possible in the therapeutic effects of an autologous tumor cell vaccine in the B16 melanoma model. The vaccine was engineered to secrete IL-12 and IL18 by means of the EBV/lipoplex. B16 cells were subcutaneously implanted into syngenic mice followed by repetitive immunization with irradiated B16 cells that had been transfected 3 days earlier by TFL2-3, a novel cationic lipid, with EBV-plasmid vectors encoding IL-12 and/or IL-18 genes (B16/mIL-12, B16/mIL-18, and B16/mIL12+mIL-18) [108]. Recombinant interleukin (IL)-2 administrations can mediate regression of solid tumors in patients with melanoma and renal cell carcinoma. A better understanding of the mechanisms of IL-2-mediated antitumor effects has led to the investigation of novel immunotherapeutic approaches. The rationale for these immunotherapeutic approaches and the results of preliminary clinical studies are presented [109]. Rapid growth in oncogenomics area used to live bacterial cells research and therapeutic purposes. Recognition, which cells can be genetically engineered to synthesize products that have therapeutic potential has generated considerable interest and excitement among clinicians and health professionals. It is expected that, a wide range of disease modifying substrates, such as hormones, antibodies, enzymes, vaccines, and other genetic products will be used successfully and will impact upon health care substantially [110]. Recent ventures in metabolic engineering and heterologous protein expression have enhanced the enzymatic and immunomodulatory effects of probiotics and, with time, may allow more active intervention among critical care patients. In addition, a number of approaches are currently being explored, including the physical and chemical protection of cells, to increase probiotic viability and its health benefits. Microencapsulation in artificial cells, builds on immobilization technologies by combining enhanced mechanical stability of the capsule membrane with improved mass transport, increased cell loading, and greater control of parameters [111].

\section{Delivery of antibodies}

Exosomes are small membrane vesicles that are released into the extracellular environment during fusion of multivesicular bodies with plasma membrane. Exosomes are secreted by various cell types including hematopoietic cells, normal epithelial cells and even some tumor cells. They are known to carry MHC class I, various costimulatory molecules and some tetraspanins. Recent studies have shown the potential of using native exosomes as immunologic stimulants [112]. Exosomes secreted by dendritic cells (DCs) contain MHC-I, MHC-II, and other accessory molecules required for antigen presentation to $\mathrm{T}$ cells. Previous studies have shown that exosome MHC-I "indirectly" loaded by adding peptides to DC cultures are immunogenic. However, analysis of peptide binding was not performed to link T-cell-stimulating activity with the amount of $\mathrm{MHC}-\mathrm{I} /$ peptide complexes on the exosomes. The optimized loading conditions and the ability to transfer both MHC-I and MHC-II antigens to APC have led to the development of exosomes as an "acellular" immunotherapy approach currently being tested in clinical trials [113]. Exosomes carry immunorelevant structures which play important roles in immune response, such as $\mathrm{MHC}$ molecules, costimulatory molecules, heat shock proteins, and naive tumor antigens. Therefore they have been suggested 
as potential vaccines [114]. Therapeutic vaccines for lymphomas have been developed to induce active and long-lasting immune responses against lymphoma capable of eradicating the tumor. Most of these vaccines use the tumor B cell idiotype (the unique variable region of the surface immunoglobulin) as a tumor-specific antigen [115]. Recombinant AAV vector (rAAV) encoding a lymphoma idiotype (Id) in a singlechain variable fragment format was found to induce an efficient anti-Id immune response upon injection in immunocompetent animals. The intensity of the immune response and the protective effect of rAAV administration in vivo were systematically compared with those elicited by simple injection of naked DNA or biolistic immunization. The results indicate that Id delivery via rAAV enhances the intensity of immune response compared with injection of naked DNA, while anti-idiotypic antibodies titres are not considerably increased compared with biolistic vaccination [116].

\section{Oncogenomics strategies}

The large majority of the cases were grade 2 tumors. Most cases (all grades) were T2 or T3, and were axillary lymph node positive. Large majority of cases with nodel metastases showed extra nodal spread. The majority of patients had NPI scores greater than 5.4 indicating poor prognosis. A novel algorithm have been developed which efficiently reduces systematic noise and allows for the detection of low-level genomic alterations. The algorithm is based on comparison of the biological relevant data to data from so-called self-self hybridizations, additional experiments which contain no biological information but contain systematic errors. The fact that W1L2 has high level (>10-fold) resistance to TS inhibitor in the absence of high YES1 expression leads to a conclusion that YES1 has no direct role in the drug resistance process. The presence of tumor cells in bone marrow or apheresis product decreases DFS (disease free survival) in patients with Stage III breast cancer who underwent high-dose chemotherapy. CD34 (+) selection does not change survivals, but it may decrease the distant metastases. RSK1/RSK2 is novel activators of YB-1, able to phosphorylate the serine 102 residue. This provides a newly described mechanism whereby $\mathrm{YB}-1$ is activated in breast cancer. This implicates the EGFR/ RSK/ YB-1 pathway as an important component of BLBC, providing an important opportunity for therapeutic intervention. The molecular heterogeneity of tumor cells, together with the pleiotropic nature of the cellular response triggered by inhibition of HDACs, poses a major challenge in the quest for determinants of resistance. Expression of YB-1 universally identifies patients at high risk across all breast cancer subtypes and in situations where more aggressive treatment may be needed. We therefore propose that YB-1 may redefine high-risk breast cancer and thereby create opportunities for individualized therapy. The P-glycoprotein is uncommonly expressed in untreated lymphomas and frequently expressed in clinically drug-resistant disease, and that chemotherapy using CVAD plus maximally tolerated doses of verapamil results in a high response rate in patients carefully selected for clinical drug resistance. P-gly expression occurs in drug-refractory B-cell neoplasms and may contribute to the development of clinical drug resistance. FN-mediated adhesion confers a survival advantage for myeloma cells acutely exposed to cytotoxic drugs by inhibiting druginduced apoptosis. This finding may explain how some cells survive initial drug exposure and eventually express classical mechanisms of drug resistance such as MDR1 overexpression. The inhibitory effect of OSU-03012 on EGFR is also observed at the mRNA and protein levels. OSU03012 ultimately inhibited the growth of BLBC in monolayer and soft agar coordinate with the induction of apoptosis using an Array-Scan VTI high-content screening system.

\section{Translational and functional oncogenomics DNA-microarray, proteomic and genome sequencing studies can define tumor specific mutations and genes the expression levels of which are upregulated/downregulated in cancer cells as compared to their non-transformed counterparts. The study highlights a novel cancer immunotherapy platform with potential for translatability to the clinic and suggests its potential usefulness for controlling metastatic disease, solid tumors of limited size, or larger tumors when combined with cytotoxic agents that reduce the number of tumor-infiltrating $T$ regulatory cells.}

\section{Therapeutic and experimental strategy \\ The results resveratrol-mediated chemoprevention of rat liver carcinogenesis is devoid of any adverse cardiovascular events. Resveratrol may be developed as a chemopreventive as well as therapeutic drug for human HCC. The mTOR-targeted therapy through usage of drugs such as everolimus may be more effective for CLA of the ovary because of its significant expression of p-mTOR. The results showed that SMANCS/Lipiodol could be delivered to the metastatic lymph node via the artery, but it could not be delivered to the metastatic lesion of the lymph node via the lymphatic route. In the patient with gastric cancer, SMANCS/Lipiodol preoperatively administered via an artery was found to remain selectively in a metastatic lymph node and an anticancer effect was histologically proved in all three of the metastatic lymph nodes. The guar gum matrix}


tablets of celecoxib showed no change either in physical appearance, drug content or in dissolution pattern after storage at 40 degrees $\mathrm{C} / \mathrm{RH} 75 \%$ for 6 months. The ability to specifically target tumor with prototype-radiolabeled or fluorescent-labeled, antibody-appended CNT constructs was encouraging and suggested further investigation of CNT as a novel delivery platform.

\section{Engineering for delivery}

Improved drug delivery systems are required for drugs currently in use to treat localized diseases of the colon. Advantages of targeting drugs specifically to the diseased colon are reduced incidence of systemic side effects, lower dose of drug, supply of the drug to the biophase only when it is required and maintenance of the drug in its intact form as close as possible to the target site. The addition of recombinant and synthetic cancer vaccines to a regimen of IL-2 can result in improved antitumor responses in both animal models and melanoma patients. Vaccine-primed, tumor-specific $T$ cells may preferentially proliferate upon administration of IL-2. The apparent lack of increase in CD8+ T-cell numbers in this setting suggests that the vaccine-primed $T$ cells functionally disappear after a transient period of activation. Preventing the disappearance of activated $\mathrm{T}$ cells upon IL-2 administration-for example, by blocking proapoptotic signals-may enhances the therapeutic effectiveness of anticancer vaccines. The studies confirm the feasibility of immunotherapeutic approaches using dendritic cells and FLT3 ligand and demonstrate their potential antitumor activity. These approaches may be effective for patients with metastatic melanoma and other solid tumors and will likely be used to improve the efficacy of IL-2-based immunotherapy.

\section{Reference}

[1] Strausberg R.L., et al. (2004) Nature, 429(6990): p. 469-474.

[2] Peeper D. and Berns A. (2006) Cell, 125(7): p. 1230-1233.

[3] Blume-Jensen, P. and T. Hunter (2001) Nature, 411(6835): p. 355-365.26.

[4] Bardelli A., et al., (2003) Science, 300(5621): p. 949-949.

[5] Samuels Y., et al., (2004) Science, 304(5670): p. 554-554.

[6] Ellen G.Feigal, Nicholas C.Dracopoli, and Jeffrey M.Trent (2005) Cancer Res 65 (19): 8587-90

[7] Minjung Kim, Joseph D.Gans, Cristina Nogueira, Audrey Wang, Ji-HyePaik, BinFeng, Cameron Brennan, William C.Hahn, Carlos Cordon-Cardo, Stephan N.Wagner, Thomas J. Flotte, Lyn M. Duncan, Scott
R. Granter and Lynda Chin (2006) Cell 125,12691281.

[8] Borbone E., Berlingieri M.T., De Bellis F., Nebbioso A., Chiappetta G., Mai A., Altucci L., Fusco A. (2009) Oncogene.

[9] Ishikawa K., Tsujimoto G. (2001) Nippon Yakurigaku Zasshi. 118(3):170-6.

[10] Kalliopi Alpantakia, Eleftherios Tsiridisa, Hans-Christoph Papeb and Peter V. Giannoudisa (2007) Injury 38 (3), 263-271.

[11] Karmen Stankov (2006) Arch Oncol 13(2):69-75.

[12] Hyun Goo Woo, Eun Sung Park, Ju-Seog Lee, Yun-Han Lee, Tsuyoshi Ishikawa, Yoon Jun Kim and Snorri S. Thorgeirsson (2009) Cancer Res 69(9):4059-66.

[13] Ivo P. Touw and Stefan J. Erkeland (2007) Molecular Therapy 13-19.

[14] Achatz M.I., Hainaut P., Ashton-Prolla P. (2009) Lancet Oncol. 10(9):920-5.

[15] Murat Cokakli, Esra Erdal, Deniz Nart, Funda Yilmaz, Ozgul Sagol, Murat Kilic, Sedat Karademir and

Nese Atabey(2009) BMC Cancer 9:65

[16] Yamamoto H., Komekado H., Kikuchi A. (2006) Dev Cell 11(2):213-223.

[17] Dittmann K., Mayer C., Kehlbach R., Rodeman H.P. (2008) Mol Cancer 12(7):69.

[18] Lu X., Kambe F., Cao X., Yamauchi M., Seo H. (2008) Exp Cell Res. 314(2):342-351.

[19] Zador E. (2008) Biochem Biophys Res Commun. 375(2):265-70.

[20] Lin J.E., Li P., Pitari G.M., Schulz S., Waldman S.A. (2009) Future Oncol. 5(4):509-22.

[21] Stratford A.L., Habibi G., Astanehe A., Jiang H., Hu K., Park E., Shadeo A., Buys T.P., Lam W., Pugh T., Marra M., Nielsen T.O., Klinge U., Mertens P.R., Aparicio S., Dunn S.E. (2007) Breast Cancer Res. 9(5):R61.

[22] Shrivastav A., Selvakumar P., Bajaj G., Lu Y., Dimmock J.R., Sharma R.K. (2005) Cell Biochem Biophys. 2005; 43(1):189-202.

[23] Munshi N.C. (2008) Hematology Am Soc Hematol Educ Program. 298-305.

[24] Qasim W., Gaspar H.B., Thrasher A.J. (2009) Gene Ther.

[25] Fujii S. (2009) Nihon Rinsho Meneki Gakkai Kaishi. 32(4):231-41.

[26] Echchgadda I., Kota S., Dela Cruz I., Sabbah A., Chang T., Harnack R., Mgbemena V., Chatterjee B., Bose S. (2009) Cancer Gene Ther.

[27] Yonemitsu Y., Ueda Y., Kinoh H., Hasegawa M. (2008) Front Biosci. 13:4953-9. 
[28] Edward J. Shillitoe (2009) Head Neck Oncol. 1: 7.

[29] Miwa A. (2007) Gan to Kagaku Ryoho. 34(13):2200-16.

[30] Gomase V.S., Tagore S., Kale K.V., Bhiwgade D.A. (2008) Oncogenomics Curr Drug Metab. $\quad 9(3): 199$ 206.

[31] Arena S., Isella C., Martini M., de Marco A. and Medico E., Bardelli A. (2007) Cancer Res. 67(18):8468-76.

[32] Touw I.P., Erkeland S.J. (2007) vMol Ther. 15(1):13-9.

[33] Di Nicolantonio F., Bardelli A. (2006) Curr Opin Oncol. 18(1):69-76.

[34] Bilke S., Chen Q.R., Whiteford C.C., Khan J. (2005) Bioinformatics. 21(7):1138-45.

[35] Glinsky G.V. (2008) J Clin Oncol. 26(17):2846-53.

[36] De Groot J.F., Gilbert M.R. (2007) Curr Opin Neurol. 20(6):712-8.

[37] Law J.H., Habibi G., Hu K., Masoudi H., Wang M.Y., Stratford A.L., Park E., Gee J.M., Finlay P., Jones H.E., Nicholson R.I., Carboni J., Gottardis M., Pollak M., Dunn S.E. (2008) Cancer Res. 68(24):10238-46.

[38] Gerrit Los, Fei Yang, Goli Samimi, Gerald Manorek, Ivelina M. Guerorguieva, Stephan Howell, Nielka van Erp, and James K. Breaux (2002) Cytometry 47:66-71.

[39] Weiguang Wang, Sharon Marsh, James Cassidy, and Howard L. McLeod (2001) Cancer Research 61, 5505-5510.

[40] Martin Monte, Marta Simonatto, Leticia Y.Peche, Debora R.Bublik, Stefania Gobessi, Marco A.Pierotti, Monica Rodolfo, and Claudio Schneider (2005) PNAS 103(30), 11160-11165.

[41] Kurtis E.Bachman, Jason Sager, Ian Cheong, Marco Catto, Alberto Bardelli, Ben Ho Park, Bert Vogelstein, Angelo Carotti, Kenneth W.Kinzler, and Christoph Lengauer (2005) Mol Cancer Ther. 4(6): 1026-30.

[42] Herrmann J.L., Rastelli L., Burgess C.E., Fernandez E.E., Rothberg B.E., Rothberg J.M., Shimkets R.A. (2001) Cancer J. 7(1):40-51.

[43] Ahmad Z., Khurshid A., Qureshi A., Idress R., Asghar N., Kayani N. (2009) Indian J Pathol Microbiol. 52(4):477-81.

[44] Feletti A., Marton E., Rossi S., Canal F., Longatti P., Billeci D. (2009) J Neurooncol.

[45] Pai M.R., Mathai A.M., Kumar S., Prabhu S. (2009) Indian J Pathol Microbiol. 52(4):537-9.

[46] Rodel R.M., Matthias C., Blomeyer B.D., Wolff H.A., Jung K., Christiansen H.
(2009) Ann Otol Rhinol Laryngol. 118(9):662-9.

[47] Arpaci F., Safali M., Ozet A., Ural A.U., Cetin T., Ozturk M., Ataergin S., Kuzhan O., Komurcu S., Ozturk B., Acikel C. (2009) J Clin Apher.

[48] Albinger-Hegyi A., Stoeckli S.J., Schmid S., Storz M., lotzova G., Probst-Hensch N.M., Rehrauer $\mathrm{H}_{\text {., }} \quad$ Tinguely $\mathrm{M}$., Moch H., Hegyi I. (2009) Int J Cancer.

[49] Brautigam E., Track C., Seewald D.H., Feichtinger J., Spiegl K., Hammer J. (2009) Strahlenther Onkol. 185(10):663-8.

[50] Lockwood W. W., Chari R., Coe B.P., Girard L., MacAulay C., Lam S., Gazdar A. F., Minna J.D. and Lam W.L. (2008) Oncogene 4615-4624.

[51] James A. L. Fenton, Guy Pratt., Andy C. Rawstron, Kathryn Sibley, Dominic Rothwell, Zoe Yates, Ann Dring, Steve J. Richards, A. John Ashcroft, Faith E. Davies, Roger G. Owen, J Anthony Child and Gareth J Morgan (2003) Oncogene 1103-1113.

[52] Valeria R. Fantin and Victoria M. Richon (2007) Clinical Cancer Research 13, 7237.

[53] Tai Young Kim., Hyun-Soon Jong., SangHyun Song., Alexandre Dimtchev., Sook-Jung Jeong., Jung Weon Lee., Tae-You Kim., Noe Kyeong Kim., Mira Jung and Yung-Jue Bang (2003) Oncogene 39433951.

[54] Douglas Grossman. and Dario C. Altieri (2001) Cancer and Metastasis 20:3-11

[55] Tito Fojo., Nick Farrell., Waldo Ortuzar., Hideyuki Tanimura., John Weinstein., Timothy G., Myers Critical Reviews in Oncology/Hematology, 53 (1), 25-34.

[56] Miller T. P., Chase E. M., Dorr R., Dalton W. S., Lam K .S., Salmon S. E. (1998) Anticancer drugs, 9(2):135-40..

[57] Dalton W.S, Grogan T.M, Meltzer P.S, Scheper R.J., Durie B.G., Taylor C.W., Miller T.P. and Salmon S.E. (1989) Journal of Clinical Oncology, 7, 415424.

[58] Lori A. Hazlehurst, Nils E. Foley, Mary C. Gleason-Guzman, Miles P. Hacker, Anne E. Cress, Lee W. Greenberger, Mariska C. De Jong and William S. Dalton (1999) Cancer Research 59, 1021-1028.

[59] Cole S.P., Bhardwaj G., Gerlach J.H., Mackie J.E., Grant C.E., Almquist K.C., Stewart A.J., Kurz E.U., Duncan A.M. and Deeley R.G. ( 1992) Science, 258 (5088), 1650-1654. 
[60] Jason S. Damiano, Anne E. Cress, Lori A. Hazlehurst, Alexander A. Shtil, and William S. Dalton (1999) Blood, 93(5), 1658-1667.

[61] Stratford A.L., Fry C.J., Desilets C., Davies A.H., Cho Y.Y., Li Y., Dong Z., Berquin I.M., Roux P.P., and Dunn SE (2008) Breast Cancer Res. 10(6):R99.

[62] Habibi G., Leung S., Law J.H., Gelmon K., Masoudi H., Turbin D., Pollak M., Nielsen T.O., Huntsman D., Dunn S.E. (2008) Breast Cancer Res. 10(5):R86.

[63] Hideshima T., Mitsiades C., Tonon G., Richardson P.G., Anderson K.C. (2007) Nat Rev Cancer. 7(8):585-98.

[64] To K., Zhao Y., Jiang H., Hu K., Wang M., Wu J., Lee C., Yokom D.W., Stratford A.L., Klinge U., Mertens P.R., Chen C.S., Bally M., Yapp D., Dunn S.E. (2007) Mol Pharmacol. 72(3):641-52.

[65] Rhodes D.R., Yu J., Shanker K., Deshpande N., Varambally R., Ghosh D., Barrette T., Pandey A., Chinnaiyan A.M. (2004) Neoplasia. 6(1):1-6.

[66] Sumathi Ramachandran., Pengbo Liu., Andrew N Young., Qiqin Yin-Goen., So Dug Lim., Noelani Laycock, Mahul B Amin., Jeffrey K. Carney, Fray F. Marshall, John A. Petros and Carlos S. Moreno (2005) Oncogene 188-198

[67] Rosell., Rafael M.D., Monzo., Mariano M.D, O'Brate., Aurora Ph.D, Taron., Miquel Ph.D (2002) Current Opinion in Oncology, 14 (2), 171-179.

[68] Medico E TRANSFOG Consortium (2008) Tumori. 94(2):172-8.

[69] Sin H.S., Huh J.W., Kim D.S., Kang D.W., Min D.S., Kim T.H., Ha H.S., Kim H.H., Lee S.Y., Kim H.S. (2006) Arch Virol. 151(10):1985-94.

[70] Arkadiusz Welman., Jane Barraclough and Caroline Dive. (2007) Translational Oncogenomics 17-33

[71] Razvi M.H., Peng D., Dar A.A., Powell S.M., Frierson H.F. Jr, Moskaluk C.A, Washington K., El-Rifai W. (2007) Genes Chromosomes Cancer. 46(10):914-28.

[72] Wu J., Lee C., Yokom D., Jiang H., Cheang M.C., Yorida E., Turbin D., Berquin I.M., Mertens PR, Iftner T., Gilks C.B., Dunn S.E. (2006) Cancer Res. 66(9):4872-9.

[73] Iervolino A., Iuliano R., Trapasso F., Viglietto G., Melillo R.M., Carlomagno F., Santoro M.,Fusco A (2006) Cancer Res. 66(12):6280-7.

[74] Furney S.J., Calvo B., Larranaga P., Lozano J.A., Lopez-Bigas N. (2008) Nucleic Acids Res. 36(18):e115.
[75] Furge K.A., Tan M.H., Dykema K., Kort E., Stadler W., Yao X., Zhou M., Teh B.T. (2007) Oncogene. 26(9):1346-50.

[76] Su W.H., Chao C.C., Yeh S.H., Chen D.S., Chen P.J., Jou Y.S. (2007) Nucleic Acids Res. 35(Database issue):D72731.

[77] Jayandharan G.R., Zhong L., Sack B.K., Rivers A.E., Li M., Li B., Herzog R., Srivastava A. (2009) Hum Gene Ther.

[78] Smith K.A., Meisenburg B.L., Tam V.L., Pagarigan R.R., Wong R., Joea D.K., Lantzy L., Carrillo MA., Gross T.M., Malyankar U.M., Chiang C.S., Da Silva D.M., Kundig T.M., Kast W.M., Qiu Z., Bot A. (2009) Clin Cancer Res. 15(19):6167-76.

[79] Fortin C.F., McDonald P.P., Fulop T., Lesur O. (2009) Shock. Colunga A.G., Laing J.M., Aurelian L. (2009) Gene Ther.

[80] Luther D.J., Ohanyan V., Shamhart P.E., Hodnichak C.M., Sisakian H., Booth T.D., Meszaros JG., Bishayee A. (2009) Invest New Drugs.

[81] Wang H.D., Lu D.X., Qi R.B. (2009) Pathophysiology. 16(4):291-6.

[82] Miyazawa M., Yasuda M., Fujita M., Kajiwara H., Hirabayashi K., Takekoshi S., Hirasawa T., Murakami M., Ogane N., Kiguchi K., Ishiwata I., Mikami M., Osamura R.Y. (2009) Pathol Int. 59(1):19-27.

[83] Jain K.K. (2004) Expert Rev Proteomics. $1(1): 49-55$.

[84] DaRue A Prieto., Xiaoying Ye and Timothy D Veenstra. (2005) Expert Review of Proteomics, 2 (4), 603-614.

[85] Andrew M. Smith., Shivang Dave., Shuming Nie., Lawrence True and Xiaohu Gao. (2006) Expert Review of Molecular Diagnostics 6 (2), 231-244.

[86] Brice P., Sanderson S. (2006) Pharmaceutical Journal 277; 113-4

[87] Golshan M., Miron A., Nixon A.J., Garber J.E., Cash E.P., iglehart J.D et.al (2006) American Journal of Surgery 192; 58-62

[88] Schlich-Bakker K.J., ten Kroode H.F.J., Ausems M. (2006) Patients Eduation and Counseling 62(1); 13-20

[89] Jain K.K. (2004) Expert Rev Proteomics. 1(1):49-55.

[90] Edison T. Liu. (2005) Briefings in Functional Genomics and Proteomics 3(4):303321.

[91] Niesner U., Halin C., Lozzi L., Gunthert M., Neri P., Wunderli-Allenspach H., Zardi L., Neri D. (2002) Bioconjug Chem. 13(4):729-36.

[92] Fleming A.B., Saltzman W.M. (2002) Clin Pharmacokinet. 41(6):403-19.

[93] Maeda H. (1989) Gan To Kagaku Ryoho. 16(10):3323-31. 
[94] Ohtsuka N., Konno T., Miyauchi Y., Maeda H. (1987) Cancer. 59(9):1560-5.

[95] Maeda H., Konno T., Iwai K., Maki S., Tashiro S. (1984) Gan To Kagaku Ryoho. 11(4):814-26.

[96] Krishnaiah Y.S., Satyanarayana V., Kumar B.D., Karthikeyan R.S. (2002) J Drug Targ. 10(3):247-54.

[97] Keler T., He L., Graziano R.F. (2005) Curr Opin Mol Ther. 7(2):157-63.

[98] Mocellin S., Lise M., Nitti D. (2005) Trends Mol Med. 11(7):327-35.

[99] Argnani R., Lufino M., Manservigi M., Manservigi R. (2005) Gene Ther. 12 Suppl 1:S170-7.

[100] Judge A., McClintock K., Phelps JR., Maclachlan I. (2006) Mol Ther. 13(2):328-37.

[101] Saito R., Kumabe T., Tominaga T. (2006) No Shinkei Geka. 34(11):1157-65.

[102] Liakatos A., Kunz H. (2007) Curr Opin Mol Ther. 9(1):35-44.

[103] McDevitt M.R., Chattopadhyay D., Kappel B.J., Jaggi J.S., Schiffman S.R., Antczak C., Njardarson J.T., Brentjens R., Scheinberg D.A. (2007) J Nucl Med. 48(7):1180-9.

[104] Hellstrom K.E., Hellstrom I. (2007) J Cell Biochem. 102(2):291-300.

[105] Chourasia M.K., Jain S.K. (2003) J Pharm Pharm Sci. 6(1):33-66.

[106] Overwijk W.W., Theoret M.R., Restifo N.P. (2000) Cancer J Sci Am. 6 Suppl 1:S7680.

[107] Asada H., Kishida T., Hirai H., Satoh E., Ohashi S., Takeuchi M., Kubo T., Kita M., Iwakura Y., Imanishi J., Mazda O. (2002) Mol Ther. 5(5 Pt 1):609-16.

[108] Lotze M.T., Shurin M., Esche C., Tahara H., Storkus W., Kirkwood J.M., Whiteside T.L., Elder EM., Okada H., Robbins P. (2000) Cancer J Sci Am. 6 Suppl 1:S616.

[109] Prakash S., Jones M.L. (2005) J Biomed Biotechnol. 2005(1):44-56.

[110] Prakash S., Martoni C. (2006) Appl Biochem Biotechnol. 128(1):1-22.

[111] Cho J.A., Yeo D.J., Son H.Y., Kim H.W., Jung D.S., Ko J.K., Koh J.S., Kim Y.N., Kim C.W. (2005) Int $J$ Cancer. 114(4):613-22.

[112] Hsu D.H., Paz P., Villaflor G., Rivas A., Mehta-Damani A., Angevin E., Zitvogel L., Le Pecq J.B. (2003) J Immunother. 26(5):440-50.

[113] Li X., Zhang Z., Beiter T., Schluesener H.J. (2005) Arch Immunol Ther Exp (Warsz). 53(4):329- 35.

[114] Houot R., Levy R. (2009) Blood Rev. 23(3):137-42.
[115] Cesco-Gaspere M., Zentilin L., Giacca M., Burrone O.R. (2008) Scand J Immunol. 68(1):58-66. 